Pacific

Journal of

Mathematics

TOPOLOGICAL DYNAMICS ON MODULI SPACES, I

Joseph P. Previte and Eugene Z. Xia 


\title{
TOPOLOGICAL DYNAMICS ON MODULI SPACES, I
}

\author{
Joseph P. Previte And Eugene Z. Xia
}

Let $M$ be a one-holed torus with boundary $\partial M$ (a circle) and $\Gamma$ the mapping class group of $M$ fixing $\partial M$. The group $\Gamma$ acts on $\mathcal{M}_{\mathcal{C}}(\mathrm{SU}(2))$ which is the space of $\mathrm{SU}(2)$-gauge equivalence classes of flat $\mathrm{SU}(2)$-connections on $M$ with fixed holonomy on $\partial M$. We study the topological dynamics of the $\Gamma$-action and give conditions for the individual $\Gamma$-orbits to be dense in $\mathcal{M}_{\mathcal{C}}(\mathrm{SU}(2))$.

\section{Introduction.}

Let $M$ be a Riemann surface of genus $g$ with $m$ boundary components (circles). Let

$$
\left\{\gamma_{1}, \gamma_{2}, \ldots, \gamma_{m}\right\} \subset \pi_{1}(M)
$$

be the elements in the fundamental group corresponding to these $m$ boundary components. The space of $\mathrm{SU}(2)$-gauge equivalence classes of $\mathrm{SU}(2)$ connections, $Y M_{2}(\mathrm{SU}(2))$, is the well-known Yang-Mills two space of quantum field theory. Inside $Y M_{2}(\mathrm{SU}(2))$ is the moduli space $\mathcal{M}(\mathrm{SU}(2))$ of flat $\mathrm{SU}(2)$-connections.

The moduli space $\mathcal{M}(\mathrm{SU}(2))$ has an interpretation that relates to the representation space $\operatorname{Hom}\left(\pi_{1}(X), \mathrm{SU}(2)\right)$ which is a real algebraic variety. The group $\mathrm{SU}(2)$ acts on $\operatorname{Hom}\left(\pi_{1}(M), \mathrm{SU}(2)\right)$ by conjugation, and the resulting quotient space is precisely

$$
\mathcal{M}(\mathrm{SU}(2))=\operatorname{Hom}\left(\pi_{1}(M), \mathrm{SU}(2)\right) / \mathrm{SU}(2) .
$$

Conceptually, the moduli space $\mathcal{M}(\mathrm{SU}(2))$ relates to the semi-classical limit of $Y M_{2}(\mathrm{SU}(2))$.

Assign each $\gamma_{i}$ a conjugacy class $C_{i} \subset \mathrm{SU}(2)$ and let

$$
\mathcal{C}=\left\{C_{1}, C_{2}, \ldots, C_{m}\right\}
$$

Definition 1.1. The relative character variety with respect to $\mathcal{C}$ is

$$
\mathcal{M}_{\mathcal{C}}(\mathrm{SU}(2))=\left\{[\rho] \in \mathcal{M}: \rho\left(\gamma_{i}\right) \in C_{i}, 1 \leq i \leq m\right\} .
$$

The space $\mathcal{M}_{\mathcal{C}}(\mathrm{SU}(2))$ is compact, but possibly singular. The set of smooth points of $\mathcal{M}_{\mathcal{C}}(\mathrm{SU}(2))$ possesses a natural symplectic structure $\omega$ which gives rise to a finite measure $\mu$ on $\mathcal{M}_{\mathcal{C}}(\mathrm{SU}(2))$ (see $[\mathbf{2}, \mathbf{3}, \mathbf{5}]$ ). 
Let $\operatorname{Diff}(M, \partial M)$ be the group of diffeomorphisms fixing $\partial M$. The mapping class group $\Gamma$ is defined to be $\pi_{0}(\operatorname{Diff}(M, \partial M))$. The group $\Gamma$ acts on $\pi_{1}(M)$ fixing the $\gamma_{i}$ 's. It is known that $\omega$ (hence $\mu$ ) is invariant with respect to the $\Gamma$-action. In [2], Goldman showed that with respect to the measure $\mu$ :

Theorem 1.2 (Goldman). The the mapping class group $\Gamma$ acts ergodically on $\mathcal{M}_{\mathcal{C}}(\mathrm{SU}(2))$.

Since $\mathcal{M}_{\mathcal{C}}(\mathrm{SU}(2))$ is a variety, one may also study the topological dynamics of the mapping class group action. The topological-dynamical problem is considerably more delicate. To begin, not all orbits are dense in $\mathcal{M}_{\mathcal{C}}(\mathrm{SU}(2))$. If $\sigma \in \operatorname{Hom}\left(\pi_{1}(M), G\right)$ where $G$ is a proper closed subgroup of $\mathrm{SU}(2)$ and $\tau \in \Gamma$, then $\tau(\sigma) \in \operatorname{Hom}\left(\pi_{1}(M), G\right)$. In other words, $\mathcal{M}_{\mathcal{C}}(G) \subset$ $\mathcal{M}_{\mathcal{C}}(\mathrm{SU}(2))$ is invariant with respect to the $\Gamma$-action. The main result of this paper is the following:

Theorem 1.3. Suppose $M$ is a torus with one boundary component and $\sigma \in \operatorname{Hom}\left(\pi_{1}(M), \mathrm{SU}(2)\right)$ such that $\sigma\left(\pi_{1}(M)\right)$ is dense in $\mathrm{SU}(2)$. Then the $\Gamma$-orbit of the conjugacy class $[\sigma] \in \mathcal{M}_{\mathcal{C}}(\mathrm{SU}(2))$ is dense in $\mathcal{M}_{\mathcal{C}}(\mathrm{SU}(2))$.

The group $\mathrm{SU}(2)$ is a double cover of $\mathrm{SO}(3)$ :

$$
p: \mathrm{SU}(2) \longrightarrow \mathrm{SO}(3) \text {. }
$$

The group $\mathrm{SO}(3)$ contains $\mathrm{O}(2)$, and the symmetry groups of the regular polyhedra: $T^{\prime}$ (the tetrahedron), $C^{\prime}$ (the cube), and $D^{\prime}$ (the dodecahedron). Let $\operatorname{Pin}(2), T, C$, and $D$ denote the groups $p^{-1}(\mathrm{O}(2)), p^{-1}\left(T^{\prime}\right), p^{-1}\left(C^{\prime}\right)$, and $p^{-1}\left(D^{\prime}\right)$, respectively. The proper closed subgroups of $\mathrm{SU}(2)$ consist of $T$, $C, D$, and the closed subgroups of $\operatorname{Pin}(2)$. In particular, $T \subset C$ and $T \subset D$. Suppose $\sigma \in \operatorname{Hom}\left(\pi_{1}(X), \mathrm{SU}(2)\right)$. Denote $[\sigma]$ the corresponding $\mathrm{SU}(2)$ conjugacy class in $\mathcal{M}_{\mathcal{C}}(\mathrm{SU}(2))$. Theorem 1.3 implies that if $\sigma\left(\pi_{1}(M)\right)$ is not contained in a group isomorphic to $C, D$, or $\operatorname{Pin}(2)$, then the $\Gamma$-orbit of the conjugacy class $[\sigma] \in \mathcal{M}_{\mathcal{C}}(\mathrm{SU}(2))$ is dense in $\mathcal{M}_{\mathcal{C}}(\mathrm{SU}(2))$.

A conjugacy class in $\mathrm{SU}(2)$ is determined by its trace. For $M$ a torus with one boundary component, the moduli space $\mathcal{M}(\mathrm{SU}(2))$ is a topological ball while $\mathcal{M}_{\mathcal{C}}(\mathrm{SU}(2))$ is generically a smooth 2 -sphere. The mapping class group $\Gamma$ is generated by two Dehn twists $\tau_{X}, \tau_{Y}$. With a proper change of coordinates, $\tau_{X}$ and $\tau_{Y}$ act on $\mathcal{M}_{\mathcal{C}}(\mathrm{SU}(2))$ by rotations along two axes. The angle of rotation depends on the latitude of the circle along the respective axis. The ergodicity theorem for $\mathcal{M}_{\mathcal{C}}(\mathrm{SU}(2))$ follows since almost all the rotations are irrational multiples of $2 \pi$. However, in the context of topological dynamics, one must analyze the orbit of each class $[\rho] \in \mathcal{M}_{\mathcal{C}}(\mathrm{SU}(2))$ upon which one or both of the $\tau_{X}, \tau_{Y}$ actions are rotations of rational multiples of $2 \pi$.

The proof of Theorem 1.3 consists of two steps. The first is purely topological-dynamical in nature, concerning the case when the $\Gamma$-orbit is 
infinite. The second step deals with the cases where the $\Gamma$-orbits are potentially finite and involves the theory of trigonometric Diophantine equations. All in all, the proof is a delicate interplay of ideas in geometric invariant theory $[6,7]$, topological dynamics, and Diophantine equations. Incidentally, the proof also yields the well-known result that the only proper closed subgroups $\mathrm{SU}(2)$ are the closed subgroups of $\mathrm{Pin}(2)$ and the double covers of the automorphism groups of the Platonic solids.

The following conjecture is the analogue of Theorem 1.2 in the category of topological dynamics. Theorem 1.3 is a major stepping stone in the search of a proof for this conjecture.

Conjecture 1.4. Suppose that $M$ is a Riemann surface with boundary and $\sigma \in \operatorname{Hom}\left(\pi_{1}(M), \mathrm{SU}(2)\right)$ such that $\sigma\left(\pi_{1}(M)\right)$ is dense in $\mathrm{SU}(2)$. Then the $\Gamma$-orbit of the conjugacy class $[\sigma] \in \mathcal{M}_{\mathcal{C}}(\mathrm{SU}(2))$ is dense in $\mathcal{M}_{\mathcal{C}}(\mathrm{SU}(2))$.

Acknowledgments. During the course of this research, Eugene Xia was with the University of Arizona. We thank Professors Michael Brin, William Goldman, Larry Grove, Kirti Joshi, David Levermore, William McCallum, Michelle Previte, and Lawrence Washington for insightful discussions during the course of this research. Eugene Xia also thanks IHÉS for their hospitality during the final phase of this research.

\section{Coordinates on the Moduli Space.}

For the rest of this paper, fix $M$ to be a torus with one boundary component. We write $E$ for $\mathcal{M}(\mathrm{SU}(2))$ and $E_{k}$ for $\mathcal{M}_{\mathcal{C}}(\mathrm{SU}(2))$ such that $k=\operatorname{tr}(C)$, where $C$ is the sole element in $\mathcal{C}$. In this section, we briefly summarize some general properties of $E$. Consult [2] for details.

The fundamental group $\pi_{1}(M)$ has a presentation

$$
\pi_{1}(M)=\left\langle X, Y, K \mid K=X Y X^{-1} Y^{-1}\right\rangle
$$

where $K$ represents the element generated by the boundary component. In particular, $\pi_{1}(M)$ is the free group generated by $X$ and $Y$. Note

$$
E=\operatorname{Hom}\left(\pi_{1}(M), \mathrm{SU}(2)\right) / \mathrm{SU}(2) .
$$

The $\mathrm{SU}(2)$-invariant polynomials $[7]$ on $\operatorname{Hom}\left(\pi_{1}(M), \mathrm{SU}(2)\right)$ are generated by the traces of the representations. In particular, a point $[\sigma] \in E$ is determined by

$$
x=\operatorname{tr}(\sigma(X)), y=\operatorname{tr}(\sigma(Y)), z=\operatorname{tr}(\sigma(X Y)) .
$$

This provides a global coordinate chart:

$$
\begin{gathered}
F: E \longmapsto \mathbb{R}^{3} \\
{[\sigma] \stackrel{F}{\longmapsto}(\operatorname{tr}(\sigma(X)), \operatorname{tr}(\sigma(Y)), \operatorname{tr}(\sigma(X Y))) .}
\end{gathered}
$$


In addition, $k=\operatorname{tr}(\sigma(K))$ is given by the formula

$$
k=\operatorname{tr}(\sigma(K))=x^{2}+y^{2}+z^{2}-x y z-2 .
$$

The trace of every element in $\mathrm{SU}(2)$ is in $[-2,2]$. In fact, one can show that

$$
E=\left\{(x, y, z) \in[-2,2]^{3}:-2 \leq k \leq 2\right\} .
$$

Let $f$ be the map

$$
\begin{gathered}
f: E \longrightarrow[-2,2] \\
f([\sigma])=\operatorname{tr}(\sigma(K)) .
\end{gathered}
$$

The fibre $f^{-1}(k)$ is precisely $E_{k}$ and is a smooth 2-sphere for each $-2<k<$ 2. The fibre $f^{-1}(2)$ is a singular sphere while $f^{-1}(-2)$ consists of one point. The mapping class group $\Gamma$ is generated by the maps $\tau_{X}$ and $\tau_{Y}$ :

$$
\begin{aligned}
& \tau_{X}(X)=X \text { and } \tau_{X}(Y)=Y X \\
& \tau_{Y}(X)=X Y \text { and } \tau_{Y}(Y)=Y .
\end{aligned}
$$

The induced action of $\Gamma$ on $E$ preserves $E_{k}$.

With respect to the global coordinate, the actions of $\tau_{X}$ and $\tau_{Y}$ can be described explicitly:

$$
\begin{aligned}
& \tau_{X}(x, y, z)=(x, z, x z-y) \\
& \tau_{Y}(x, y, z)=(z, y, y z-x) .
\end{aligned}
$$

The action of $\tau_{X}$ fixes $x$ and $k$, and preserves the ellipse

$$
E_{x, k}=\{x\} \times\left\{(y, z): \frac{2+x}{4}(y+z)^{2}+\frac{2-x}{4}(y-z)^{2}=2+k-x^{2}\right\} .
$$

The topological sphere $f^{-1}(1)$ is pictured below, decomposed into ellipses.

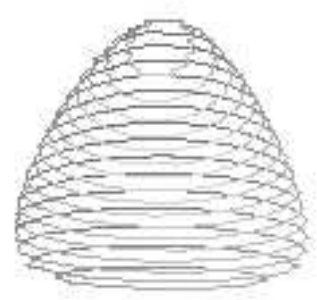

Figure 1. The topological sphere $E_{1}$.

The change of coordinates

$$
\left\{\begin{array}{l}
\tilde{x}=x \\
\tilde{y}=\frac{\sqrt{2-x}+\sqrt{2+x}}{2 \sqrt{2}} y+\frac{\sqrt{2-x}-\sqrt{2+x}}{2 \sqrt{2}} z \\
\tilde{z}=\frac{\sqrt{2-x}-\sqrt{2+x}}{2 \sqrt{2}} y+\frac{\sqrt{2-x}+\sqrt{2+x}}{2 \sqrt{2}} z
\end{array}\right.
$$


transforms $E_{x, k}$ into the circle

$$
E_{x, k}=\{\tilde{x}\} \times\left\{(\tilde{y}, \tilde{z}): \tilde{y}^{2}+\tilde{z}^{2}=2+k-\tilde{x}^{2}\right\} .
$$

In this new coordinate system, $\tau_{X}$ acts as a rotation by $\cos ^{-1}(x / 2)$. In short, the sphere $E_{k}$ is the union of circles

$$
E_{k}=\bigcup_{x} E_{x, k},
$$

and $\tau_{X}$ rotates (up to a coordinate transformation) each level set $E_{x, k}$ by an angle of $\cos ^{-1}(x / 2)$.

Under an analogous coordinate transformation, the action $\tau_{Y}$ becomes a rotation of $E_{y, k}$ by an angle of $\cos ^{-1}(y / 2)$.

\section{The Closed Subgroup Representations.}

All closed proper subgroups of SU(2) are contained in $\mathrm{Pin}(2), C$, or $D$, where $C$ and $D$ are the double covers of the isometry groups of the cube and dodecahedron, respectively. In this section, we classify the Pin(2) representation classes and produce a list of global coordinates for some $C, D$ representation classes. We shall prove later that the list is complete up to some simplifying assumptions and the variations allowed by the following proposition:

Proposition 3.1. Suppose $\sigma$ is a $G$ representation such that $G \subset \mathrm{SU}(2)$. If $(x, y, z)$ are the global coordinates of $[\sigma]$, then any permutation of $(x, y, z)$ also corresponds to a $G$ representation class. In addition, if $-I \in G$, then the triples $(-x, y,-z),(x,-y,-z),(-x,-y, z)$ also correspond to $G$ representation classes.

Proof. Suppose $\sigma$ is a $G$ representation such that $[\sigma]$ has global coordinates $(x, y, z)$. Since $G$ is a group, the representation $\sigma^{\prime}$, with

$$
\sigma^{\prime}(X)=\sigma(X Y), \quad \sigma^{\prime}(Y)=\sigma\left(X^{-1}\right),
$$

is also a $G$ representation. Moreover $\left[\sigma^{\prime}\right]$ has global coordinates $(z, x, y)$. The other permutations of coordinates are handled similarly.

Suppose $\sigma$ is a $G$ representation. Since $-I \in G$, the representation $\sigma^{\prime}$, with

$$
\sigma^{\prime}(X)=-\sigma(X), \quad \sigma^{\prime}(Y)=\sigma(Y)
$$

is also a $G$ representation and the global coordinates of $\left[\sigma^{\prime}\right]$ is $(-x, y,-z)$. The other cases are handled similarly.

If $-I \in G$, then two classes

$$
[\sigma],\left[\sigma^{\prime}\right] \in \operatorname{Hom}\left(\pi_{1}(M), G\right) / G
$$

are called $S$-equivalent if their global coordinates differ from one another as prescribed by Proposition 3.1. If $-I$ is not in $G$, then two classes $[\sigma],\left[\sigma^{\prime}\right]$ 
are called $S$-equivalent if their global coordinates differ from one another by a permutation.

3.1. The Pin(2) Representations. The group Pin(2) has two components, and we write

$$
\operatorname{Pin}(2)=\operatorname{Spin}(2) \cup \operatorname{Spin}_{-}(2),
$$

where $\operatorname{Spin}(2)$ is the identity component of $\operatorname{Pin}(2)$.

Proposition 3.2. A representation $\sigma$ is a $\operatorname{Spin}(2)$ representation if and only if $\operatorname{tr}(\sigma(K))=2$.

Proof. If $\sigma$ is a $\operatorname{Spin}(2)$ representation, then

$$
\sigma(K)=\sigma\left(X Y X^{-1} Y^{-1}\right)=I,
$$

since $\operatorname{Spin}(2)$ is abelian. Hence,

$$
k=\operatorname{tr}(\sigma(K))=\operatorname{tr}(I)=2 .
$$

If $k=\operatorname{tr}(\sigma(K))=2$, then

$$
I=\sigma(K)=\sigma(X) \sigma(Y) \sigma(X)^{-1} \sigma(Y)^{-1} .
$$

This implies that the image of $\sigma$ is abelian, hence, is contained in $\operatorname{Spin}(2)$.

Proposition 3.3. A representation $\sigma$ is a $\operatorname{Pin}(2)$ representation and not a $\operatorname{Spin}(2)$ representation if and only if $k \neq 2$ and at least two of the three global coordinates of $[\sigma]$ are zero.

Proof. If $\sigma$ is a $\operatorname{Pin}(2)$ representation and not a $\operatorname{Spin}(2)$ representation, then at least two of the following

$$
\sigma(X), \sigma(Y), \sigma(X Y)
$$

are in $\operatorname{Spin}_{-}(2)$. Since $A \in \operatorname{Spin}_{-}(2)$ implies $\operatorname{tr}(A)=0$, at least two of the three global coordinates of $[\sigma]$ must be zero.

Suppose two of the three global coordinates of $[\sigma]$ are zero, say $y, z=0$. One easily finds a $\operatorname{Pin}(2)$ representation $\sigma^{\prime}$ such that the global coordinates of $\left[\sigma^{\prime}\right]$ are $(x, 0,0)$ :

$$
\sigma^{\prime}(X)=\left(\begin{array}{cc}
\cos (\theta) & \sin (\theta) \\
-\sin (\theta) & \cos (\theta)
\end{array}\right), \sigma^{\prime}(Y)=\left(\begin{array}{ll}
0 & i \\
i & 0
\end{array}\right),
$$

where $x=2 \cos (\theta)$. If $x \neq \pm 2$, then $k \neq 2$ and $\sigma$ is not a $\operatorname{Spin}(2)$ representation. Since global coordinates are unique,

$$
[\sigma]=\left[\sigma^{\prime}\right] \in \operatorname{Hom}\left(\pi_{1}(M), \mathrm{SU}(2)\right) / \mathrm{SU}(2) .
$$

Thus, $\sigma$ is a $\operatorname{Pin}(2)$ representation but not a $\operatorname{Spin}(2)$ representation. The proofs for the other cases are similar. 
This provides a complete characterization of the $\operatorname{Pin}(2)$ representation classes.

Corollary 3.4. The space of $\operatorname{Spin}(2)$ representation classes consists precisely of $E_{2}$ (or $\left.\partial E\right)$. The $\operatorname{Pin}(2)$ representation classes consist of $E_{2}$ and the intersections of the three axes with $E$. For $-2<k<2$, there are exactly six points corresponding to $\mathrm{Pin}(2)$ representation classes in $E_{k}$.

3.2. The $C$ and $D$ Representations. Since $C$ (respectively, $D$ ) is finite, the $\Gamma$-orbit of a $C$ (respectively, $D$ ) representation class is finite. One also notes that $-I$ is in $C$ and $D$.

We introduce the quaternionic model for $\mathrm{SU}(2)$, namely set $1, \mathrm{i}, \mathrm{j}, \mathrm{k}$ as

$$
\left[\begin{array}{ll}
1 & 0 \\
0 & 1
\end{array}\right], \quad\left[\begin{array}{cc}
i & 0 \\
0 & -i
\end{array}\right], \quad\left[\begin{array}{cc}
0 & 1 \\
-1 & 0
\end{array}\right], \quad\left[\begin{array}{ll}
0 & i \\
i & 0
\end{array}\right]
$$

respectively. Then $\mathrm{SU}(2)=\left\{x+y \mathrm{i}+z \mathrm{j}+w \mathrm{k}: x^{2}+y^{2}+z^{2}+w^{2}=1\right\}$, with the usual quaternionic multiplication.

For the rest of the paper, we fix the constants

$$
\left\{\begin{array}{l}
r=\frac{\sqrt{5}+1}{4} \\
s=\frac{\sqrt{5}-1}{4} .
\end{array}\right.
$$

Let

$$
\begin{aligned}
& \left\{\begin{array}{l}
T=\frac{\sqrt{2}}{2}+\frac{\sqrt{2}}{2} \mathrm{i} \\
U=\frac{\sqrt{2}}{2}+\frac{\sqrt{2}}{2} \mathrm{j},
\end{array}\right. \\
& \left\{\begin{array}{l}
A=r+s \mathrm{i}+\frac{1}{2} \mathrm{k} \\
B=-s+\frac{1}{2} \mathrm{i}-r \mathrm{k} .
\end{array}\right.
\end{aligned}
$$

Then

$$
\langle T, U\rangle \cong C \text { and }\langle A, B\rangle \cong D
$$




\begin{tabular}{|c|c|c|c|}
\hline$X$ & $Y$ & $(x, y, z)$ & $k$ \\
\hline$T$ & $(T U)^{-1}$ & $(\sqrt{2}, 1, \sqrt{2})$ & 1 \\
$A^{3} B^{8}$ & $(A A B A)^{-1}$ & $(1,1,1)$ & 0 \\
$B^{-1}$ & $A A B$ & $(-2 s, 2 s, 2 s)$ & $\frac{1-\sqrt{5}}{2}$ \\
$A B A^{3} B^{2}$ & $B$ & $(-2 s,-2 s, 1)$ & $\frac{1-\sqrt{5}}{2}$ \\
$A^{-1}$ & $A B B$ & $(2 r,-2 r,-2 r)$ & $\frac{1+\sqrt{5}}{2}$ \\
$A B A A B^{6}$ & $A A B A$ & $(2 r, 1,2 r)$ & $\frac{1+\sqrt{5}}{2}$ \\
$A B A A$ & $A$ & $(1,2 r, 1)$ & 1 \\
$A B^{-6}$ & $B^{-6}$ & $(1,2 s,-1)$ & 1 \\
$A B A A$ & $A^{-1}$ & $(1,2 r, 2 s)$ & 1 \\
\hline
\end{tabular}

Table 1. Global coordinates for some $C$ and $D$ representation classes.

Table 1 is a list of $S$-equivalence classes that come from either $\langle T, U\rangle$ (cube) or $\langle A, B\rangle$ (dodecahedron) representation classes. We shall prove in Section 6 the following statement. Suppose $(x, y, z)$ is a $C$ or $D$ representation class, but not a $\operatorname{Pin}(2)$ representation class. Then there exists $\gamma \in \Gamma$ such that $\gamma(x, y, z)$ is in one of the $S$-equivalence classes in Table 1.

\section{The Irrational Rotations and Infinite Orbits.}

The Dehn twist $\tau_{Y}$ acts on the (transformed) subsets $E_{y, k}$ via a rotation of angle $\cos ^{-1}(y / 2)$. The $y$-coordinates that yield finite orbits under $\tau_{Y}$ create a filtration as follows: Let $Y_{n} \subset(-2,2)$ such that $y \in Y_{n}$ implies that if $(x, y, z) \in E$ are global coordinates of a representation class, then the $\tau_{Y^{-}}$ orbit of $(x, y, z)$ is periodic with period greater than one but less than or equal to $n$. This gives a filtration

$$
\emptyset=Y_{2} \subset Y_{3} \subset \ldots \subset Y_{n} \subset \ldots
$$

For example, $Y_{2}=\emptyset, Y_{3}=\{-1\}, Y_{4}=\{-1,0\}$, etc. In particular, $Y_{n}$ is a finite set for every $n$. By symmetry, there exists a similar filtration $X_{n}$, with $X_{n}=Y_{n}$ as sets.

Fix $-2<k<2$ and consider the two-dimensional sphere $E_{k}$. The global coordinates provide an embedding of $E_{k}$ in $\mathbb{R}^{3}$ as a submanifold. Hence $E_{k}$ inherits a metric from the flat Riemannian metric on $\mathbb{R}^{3}$. This provides a distance function (metric) $d$ on $E_{k}$. The metric $d$ generates the usual topology on $E_{k}$. Note that there are two points on $E_{k}$ that are fixed by $\tau_{Y}$. These points correspond to $\operatorname{Pin}(2)$ representation classes. 
Definition 4.1. For $\epsilon>0$, a set $U$ is $\epsilon$-dense if for each $p \in U \subset E_{k}$, there exists a point $q \in U$ such that $0<d(p, q)<\epsilon$.

Let $\epsilon>0$. Since $E_{k}$ is compact, there is an $M(\epsilon)$ such that $n \geq M(\epsilon)$ implies that every $\tau_{Y}$-orbit $\mathcal{O}_{y}$ in $E_{y, k}$ is $\epsilon$-dense for any $y \notin Y_{M(\epsilon)}$. Let $N(\epsilon)$ be the cardinality of $Y_{M(\epsilon)}$.

Proposition 4.2. Let $\left(x_{0}, y_{0}, z_{0}\right) \in E_{k}$. Suppose that one of $\cos ^{-1}\left(x_{0} / 2\right)$ or $\cos ^{-1}\left(y_{0} / 2\right)$ is an irrational multiple of $\pi$. Then the $\Gamma$-orbit of $\left(x_{0}, y_{0}, z_{0}\right)$ is dense in $E_{k}$.

Proof. Suppose that $\cos ^{-1}\left(x_{0} / 2\right)$ is an irrational multiple of $\pi$. Let $\epsilon>0$ and $\left(x_{*}, y_{*}, z_{*}\right) \in E_{k}$ which does not correspond to a $\operatorname{Pin}(2)$ representation class. Since $\cos ^{-1}\left(x_{0} / 2\right)$ is an irrational multiple of $\pi, \tau_{X}$ acts on the (transformed) subset of $E_{x_{0}, k}$, by an irrational rotation. By the compactness of $E_{k}$, there exists a $y$-value, $y_{1} \neq y_{*}$ and $\delta>0$ such that $E_{y_{*}, k}$ is in the $\epsilon$-neighborhood of $E_{y_{1}, k}$ and $0<\delta<d\left(E_{y_{1}, k}, E_{y_{*}, k}\right)$. We first consider the special case where there exists an integer $J$ such that the $y$-coordinate of $\tau_{X}^{J}\left(x_{0}, y_{0}, z_{0}\right)$ is strictly between $y_{1}$ and $y_{*}$. Since $\cos ^{-1}\left(x_{0} / 2\right)$ is an irrational multiple of $\pi$, there are infinitely many integers $J_{i}$ such that the $y$-coordinate of $\tau_{X}^{J_{i}}\left(x_{0}, y_{0}, z_{0}\right)$ is strictly between $y_{1}$ and $y_{*}$. Choose $J_{i}$ such that the $y$ coordinate of $\left(\tau_{X}\right)^{J_{i}}\left(x_{0}, y_{0}, z_{0}\right)$ is not in $Y_{M(\epsilon)}$. By the triangle inequality, there is some point on the $\tau_{Y}$-orbit of $\left(\tau_{X}\right)^{J_{i}}\left(x_{0}, y_{0}, z_{0}\right)$ that is at most $2 \epsilon$ from $\left(x_{*}, y_{*}, z_{*}\right)$.

We now prove the proposition in general. Since $\left(x_{*}, y_{*}, z_{*}\right)$ satisfies Equation (1) and is not a $\operatorname{Pin}(2)$ representation class, $E_{y_{*}, k}$ is a circle. Hence, we must have that $\left(0, y_{*}, z^{\prime}\right) \in E_{y_{*}, k}$ for some $z^{\prime} \neq 0$. Therefore, there exists $x_{2} \neq 0$ such that $E_{x_{2}, k}$ intersects $E_{y_{*}, k}$.

Choose $\epsilon^{\prime}=\frac{\delta}{N(\epsilon)+2}$. By the filtration $X_{n}$, the set $X_{M\left(\epsilon^{\prime}\right)}$ contains all $x$-values that have the following properties:

1) $E_{x, k}$ intersects $E_{y_{*}, k}$.

2) There is a point in $E_{x, k}$ whose $\tau_{X}$-orbit has at most $N(\epsilon)$ points with distinct $y$-coordinates between $y_{1}$ and $y_{*}$.

Note that the $x$-coordinate of $\tau_{Y}\left(\tau_{X}\right)^{J}\left(x_{0}, y_{0}, z_{0}\right)$ is the $z$-coordinate of $\left(\tau_{X}\right)^{J}\left(x_{0}, y_{0}, z_{0}\right)$. Since $\cos ^{-1}\left(x_{0} / 2\right)$ is an irrational multiple of $\pi$, there is an infinite sequence of numbers $J_{i}$ such that $\left|x_{J_{i}}\right|<\left|x_{2}\right|$, where $x_{J_{i}}$ is the the $x$-coordinate of $\tau_{Y}\left(\tau_{X}\right)^{J_{i}}\left(x_{0}, y_{0}, z_{0}\right)$. This forces $E_{x_{J_{i}}, k}$ to intersect $E_{y_{*}, k}$. Of these, choose $J$ such that $x_{J}$ is not in $X_{M\left(\epsilon^{\prime}\right)}$. Thus, the $\tau_{X}$-orbit of $\tau_{Y}\left(\tau_{X}\right)^{J}\left(x_{0}, y_{0}, z_{0}\right)$ has at least $N(\epsilon)+1$ points with distinct $y$-coordinates between $y_{1}$ and $y_{*}$.

Now at most $N(\epsilon)$ values of $y$ yield $\tau_{Y}$-orbits that are not $\epsilon$-dense. Thus, there exists a point $(\hat{x}, \hat{y}, \hat{z})$ on the $\tau_{X}$-orbit of $\tau_{Y}\left(\tau_{X}\right)^{J}\left(x_{0}, y_{0}, z_{0}\right)$ such that $\hat{y}$ is between $y_{1}$ and $y_{*}$, moreover, the $\tau_{Y}$-orbit of $(\hat{x}, \hat{y}, \hat{z})$ is $\epsilon$-dense. Since the 
$\epsilon$-neighborhood of $E_{\hat{y}, k}$ covers $E_{y_{*}, k}$, some point in the $\tau_{Y}$-orbit of $(\hat{x}, \hat{y}, \hat{z})$ comes within $2 \epsilon$ of $\left(x_{*}, y_{*}, z_{*}\right)$. Finally, by Corollary 3.4, the set of $\operatorname{Pin}(2)$ representation classes in $E_{k}$ consists of six discrete points. This implies there is no loss of generality in assuming that $\left(x_{*}, y_{*}, z_{*}\right)$ does not correspond to a $\operatorname{Pin}(2)$ representation class. A symmetric argument holds if $\cos ^{-1}\left(y_{0} / 2\right)$ is an irrational multiple of $\pi$.

Proposition 4.3. Suppose the $\Gamma$-orbit of $\left(x_{0}, y_{0}, z_{0}\right) \in E_{k}$ is infinite. Then the $\Gamma$-orbit of $\left(x_{0}, y_{0}, z_{0}\right)$ is dense in $E_{k}$.

Proof. Let $\left(x_{0}, y_{0}, z_{0}\right) \in E_{k}$ have infinite $\Gamma$-orbit and $\left(x_{*}, y_{*}, z_{*}\right) \in E_{k}$ which does not correspond to a $\operatorname{Pin}(2)$ representation class.

There are two cases. One possibility is that the $\Gamma$-orbit $\mathcal{O}$ has an infinite number of points on some circle $E_{y, k}$ (respectively, $E_{x, k}$ ). Hence, there is an infinite number of points on $\mathcal{O} \cap E_{y, k}$ that have distinct $x$-coordinates. However, a priori, these points may not be dense in $E_{y, k}$. One uses the infinite number of points on $\mathcal{O} \cap E_{y, k}$ with distinct $x$-coordinates as in the proof of Proposition 4.2.

The other possible case is that no circle $E_{x, k}$ (or $E_{y, k}$ ) has an infinite number of points on $\mathcal{O}$. As in the previous case, there are an infinite number of points on the $\Gamma$-orbit of $\left(x_{0}, y_{0}, z_{0}\right)$ having distinct $x$-coordinates. The proof again follows similarly to the proof of Proposition 4.2.

\section{Trigonometric Diophantine Equations.}

It remains for us to classify the finite $\Gamma$-orbits. These orbits exist and can be constructed by taking $G$-representation classes where $G$ is a closed proper subgroup of $\mathrm{SU}(2)$ as described in Section 3.

The problem amounts to considering cases where the rotations generated by $\tau_{X}$ and $\tau_{Y}$ are both rational. In such cases, an additional iteration is made as follows. By assumption, both $\cos ^{-1}(x / 2)$ and $\cos ^{-1}(y / 2)$ are rational multiples of $\pi$. Also, $\cos ^{-1}(z / 2)$ is a rational multiple of $\pi$ since the $x$ coordinate of $\tau_{Y}(x, y, z)$ is $z$. Since $\tau_{X}(x, y, z)=(x, z, x z-y)$, in order for the orbit to be finite, $\cos ^{-1}\left(\frac{x z-y}{2}\right)$ must be a rational multiple of $\pi$. In particular, $x=2 \cos \left(\theta_{x}\right), y=2 \cos \left(\theta_{y}\right), z=2 \cos \left(\theta_{z}\right)$ and $x z-y=2 \cos \left(\theta_{x z-y}\right)$, where all angles are rational multiples of $\pi$ in $[0, \pi]$. Hence,

$$
2 \cos \left(\theta_{x}\right) \cos \left(\theta_{z}\right)-\cos \left(\theta_{y}\right)=\cos \left(\theta_{x z-y}\right)
$$

or

$$
\cos \left(\theta_{x}+\theta_{z}\right)+\cos \left(\theta_{x}-\theta_{z}\right)-\cos \left(\theta_{y}\right)=\cos \left(\theta_{x z-y}\right),
$$

where all angles are rational multiples of $\pi, 0 \leq \theta_{x}+\theta_{z} \leq 2 \pi$, and $-\pi \leq$ $\theta_{x}-\theta_{z} \leq \pi$. Similarly, the action of $\tau_{Y}$ gives

$$
\cos \left(\theta_{y}+\theta_{z}\right)+\cos \left(\theta_{y}-\theta_{z}\right)-\cos \left(\theta_{x}\right)=\cos \left(\theta_{y z-x}\right),
$$


where all angles are rational multiples of $\pi, 0 \leq \theta_{y}+\theta_{z} \leq 2 \pi$, and $-\pi \leq$ $\theta_{y}-\theta_{z} \leq \pi$. Equations (2) and (3) are referred to as the $\tau_{X}$-equation and $\tau_{Y}$-equation at $(x, y, z)$ (or at $\left.\left(\theta_{x}, \theta_{y}, \theta_{z}\right)\right)$, respectively.

Proposition 5.1. Let $(x, y, z) \in E_{k}$ with $x, y, z$ all nonzero. Suppose that two terms appearing in Equation (2) cancel one another. Then $k=2$.

Proof. Suppose $\cos \left(\theta_{y}\right)=-\cos \left(\theta_{x z-y}\right)$. Then $x z-y=-y$ which implies that $x=0$ or $z=0$, a contradiction to the assumption that $x, y, z$ are all nonzero.

Suppose $\cos \left(\theta_{y}\right)=\cos \left(\theta_{x}+\theta_{z}\right)$. Recall that $(x, y, z)$ satisfies Equation (1). Thus,

$$
\begin{aligned}
k= & 4 \cos \left(\theta_{x}\right)^{2}+4 \cos \left(\theta_{x}+\theta_{z}\right)^{2}+4 \cos \left(\theta_{z}\right)^{2} \\
& -8 \cos \left(\theta_{x}\right) \cos \left(\theta_{x}+\theta_{z}\right) \cos \left(\theta_{z}\right)-2 \\
= & 4 \cos \left(\theta_{x}\right)^{2}+4 \cos \left(\theta_{z}\right)^{2}-4 \cos \left(\theta_{x}-\theta_{z}\right) \cos \left(\theta_{x}+\theta_{z}\right) \\
= & 2 \cos \left(2 \theta_{x}\right)+2+2 \cos \left(2 \theta_{z}\right)-2 \cos \left(2 \theta_{x}\right)-2 \cos \left(2 \theta_{z}\right)=2 .
\end{aligned}
$$

A similar argument applies in the case $\cos \left(\theta_{y}\right)=\cos \left(\theta_{x}-\theta_{z}\right)$.

A symmetric argument shows that if two terms appearing in Equation (3) cancel one another, then $k=2$. For the remainder of this paper, we assume that $(x, y, z) \in E$ does not correspond to a $\operatorname{Pin}(2)$ representation class. This implies that $k \neq 2$. In addition, we may assume that all coordinates of $(x, y, z) \in E_{k}$ are nonzero: For if $x=0$, then $y, z$ must both be nonzero by Proposition 3.3. The point $\tau_{Y}(0, y, z)=(z, y, y z)$ has all nonzero entries.

Equation (2) is an at most four-term Diophantine equation, the solutions to which are few as shown by Conway and Jones.

Theorem 5.2 (Conway, Jones, [1]). Suppose that we have at most four distinct rational multiples of $\pi$ lying strictly between 0 and $\pi / 2$ for which some linear combination of their cosines is rational, but no proper subset has this property. That is,

$$
A \cos (a)+B \cos (b)+C \cos (c)+D \cos (d)=E,
$$

for $A, B, C, D, E$ rational and $a, b, c, d \in(0, \pi / 2)$ rational multiples of $\pi$. Then the appropriate linear combination is proportional to one from the 
following list:

$$
\begin{aligned}
\cos (\pi / 3) & =1 / 2 \\
\cos (t+\pi / 3)+\cos (\pi / 3-t)-\cos (t) & =0 \quad(0<t<\pi / 6) \\
\cos (\pi / 5)-\cos (2 \pi / 5) & =1 / 2 \\
\cos (\pi / 7)-\cos (2 \pi / 7)+\cos (3 \pi / 7) & =1 / 2 \\
\cos (\pi / 5)-\cos (\pi / 15)+\cos (4 \pi / 15) & =1 / 2 \\
-\cos (2 \pi / 5)+\cos (2 \pi / 15)-\cos (7 \pi / 15) & =1 / 2 \\
\cos (\pi / 7)+\cos (3 \pi / 7)-\cos (\pi / 21)+\cos (8 \pi / 21) & =1 / 2 \\
\cos (\pi / 7)-\cos (2 \pi / 7)+\cos (2 \pi / 21)-\cos (5 \pi / 21) & =1 / 2 \\
-\cos (2 \pi / 7)+\cos (3 \pi / 7)+\cos (4 \pi / 21)+\cos (10 \pi / 21) & =1 / 2 \\
-\cos (\pi / 15)+\cos (2 \pi / 15)+\cos (4 \pi / 15)-\cos (7 \pi / 15) & =1 / 2 .
\end{aligned}
$$

The nonzero cosine terms in Equation (2) are not necessarily in $(0, \pi / 2)$. By applying the identities $\cos (\pi / 2-t)=-\cos (\pi / 2+t)$ and $\cos (\pi-t)=$ $\cos (\pi+t)$, we derive from Equation (2) a new four-term cosine equation whose arguments are in $[0, \pi / 2]$. That is, by a possible change of sign, each term in Equation (2) may be rewritten with angle in $[0, \pi / 2]$.

Equation (2) cannot correspond to the last four equations appearing in Theorem 5.2 since these equations have five nonzero terms. By Proposition 5.1, we may assume that the angles appearing in Equation (2) are all distinct. For if two or more angles are the same, then after combining terms, the resulting equation must be proportional to the first equation in Theorem 5.2. This leads to $k=2$ by Proposition 5.1.

Proposition 5.3. Suppose $(x, y, z) \in E_{k}$ are not the global coordinates of a $\operatorname{Pin}(2)$ representation class with $x, y, z$ all nonzero. Suppose that the $\Gamma$-orbit of $(x, y, z)$ is finite and that some angle in Equation (2) or Equation (3) is an integer multiple of $\pi$. Then $(x, y, z)$ is S-equivalent to a triple appearing in Table 1.

Proof. By the assumption $k \neq 2$, the only way that Equation (2) can have angles equal to an integer multiple of $\pi$ is if $\theta_{x}-\theta_{z}=0$ or $\theta_{x}+\theta_{z}=\pi$, i.e., $x=z$ or $x=-z$. Note that both cannot happen simultaneously.

Suppose $\theta_{x}+\theta_{z}=\pi$. Then Equation (2) becomes

$$
\cos \left(\theta_{x}-\theta_{z}\right)-\cos \left(\theta_{y}\right)-\cos \left(\theta_{x z-y}\right)=1 .
$$

Theorem 5.2 and the assumption $y \neq 0$ lead to the following cases:

(A) Two terms in Equation (4) correspond to the first equation of Theorem 5.2 , with one term equal to zero (angle $\pi / 2$ ).

Since $y \neq 0$, we must have that $\cos \left(\theta_{y}\right)=-\frac{1}{2}$, so $y=-1$. Now either $\cos \left(\theta_{x z-y}\right)=0$ or $-\frac{1}{2}$. If $\cos \left(\theta_{x z-y}\right)=0$, then $x z=-1$, hence $x^{2}=1$ which yields the triples $(1,-1,-1)$ and $(-1,-1,1)$. If $\cos \left(\theta_{x z-y}\right)=\frac{1}{2}$, then 
$x z=-2$, or $x= \pm \sqrt{2}$. The resulting triples $(x, y, z)$ are $(\sqrt{2},-1,-\sqrt{2})$ and $(-\sqrt{2},-1, \sqrt{2})$. Note that all of the above triples belong to an $S$-equivalence class appearing in Table 1.

(B) Two terms in Equation (4) correspond to the third equation of Theorem 5.2 while the remaining term corresponds to the first equation in Theorem 5.2 . The resulting triples are:

$$
\begin{gathered}
(-2 s, 2 s, 2 s),(2 s, 2 s,-2 s),(-2 s,-1,2 s),(2 s,-1,-2 s), \\
(-2 r,-2 r, 2 r),(2 r,-2 r,-2 r),(-2 r,-1,2 r),(2 r,-1,-2 r), \\
(-1,-2 r, 1),(1,-2 r,-1),(-1,2 s, 1),(1,2 s,-1) .
\end{gathered}
$$

Note that all above triples belong to an $S$-equivalence class appearing in Table 1.

Suppose $\theta_{x}-\theta_{z}=0$. Then Equation (2) becomes

$$
\cos \left(\theta_{x}+\theta_{z}\right)-\cos \left(\theta_{y}\right)-\cos \left(\theta_{x z-y}\right)=-1 .
$$

Theorem 5.2 and the assumption $y \neq 0$ lead to the following cases.

(A) Two terms in Equation (5) correspond to the first equation of Theorem 5.2 , with one term equal to zero (angle $\pi / 2$ ). The various possibilities lead to the triples:

$$
(1,1,1),(-1,1,-1),(\sqrt{2}, 1, \sqrt{2}),(-\sqrt{2}, 1,-\sqrt{2}) .
$$

(B) Two terms in Equation (5) correspond to the third equation of Theorem 5.2 while the other corresponds to the first equation. The various possibilities lead to the triples:

$$
\begin{gathered}
(2 r, 2 r, 2 r),(-2 r, 2 r,-2 r),(2 r, 1,2 r),(-2 r, 1,-2 r), \\
(1,2 r, 1),(-1,2 r,-1),(1,-2 s, 1),(-1,-2 s,-1), \\
(2 s,-2 s, 2 s),(-2 s,-2 s,-2 s),(2 s, 1,2 s),(-2 s, 1,-2 s) .
\end{gathered}
$$

Again, the $S$-equivalence classes of these triples appear in Table 1. A similar argument holds if some angle in Equation (3) is an integer multiple of $\pi$, i.e., $y= \pm z$.

Henceforth, we assume that all angles in nonzero cosine terms appearing in Equation (2) are distinct and not integer multiples of $\pi$. Under these assumptions, Equation (2) can be rewritten as an equation that satisfies the hypotheses of the following which is a special case of Theorem 5.2:

Theorem 5.4 (Conway, Jones, [1]). Suppose that we have at most four distinct rational multiples of $\pi$ lying strictly between 0 and $\pi / 2$ for which some linear combination of their cosines is zero, but no proper subset has this property. That is,

$$
A \cos (a)+B \cos (b)+C \cos (c)+D \cos (d)=0,
$$


for $A, B, C, D$ rational and $a, b, c, d \in(0, \pi / 2)$ rational multiples of $\pi$. Then the linear combination is proportional to one from the following list:

$$
\begin{aligned}
\cos (t+\pi / 3)+\cos (\pi / 3-t)-\cos (t) & =0 \quad(0<t<\pi / 6) \\
\cos (\pi / 5)-\cos (2 \pi / 5)-\cos (\pi / 3) & =0 \\
\cos (\pi / 7)-\cos (2 \pi / 7)+\cos (3 \pi / 7)-\cos (\pi / 3) & =0 \\
\cos (\pi / 5)-\cos (\pi / 15)+\cos (4 \pi / 15)-\cos (\pi / 3) & =0 \\
-\cos (2 \pi / 5)+\cos (2 \pi / 15)-\cos (7 \pi / 15)-\cos (\pi / 3) & =0 .
\end{aligned}
$$

\section{Proof of Theorem 1.3.}

In this section we prove the following proposition which in turn proves Theorem 1.3:

Proposition 6.1. Let $(x, y, z) \in E_{k}$ with $x, y, z$ nonzero. Then the $\Gamma$-orbit of $(x, y, z)$ is infinite or there is $\gamma \in \Gamma$ such that $\gamma(x, y, z)$ is $S$-equivalent to a triple in Table 1.

An immediate consequence of Proposition 6.1 is:

Corollary 6.2. Suppose $(x, y, z)$ is a $C$ or $D$ representation class, but not $a \operatorname{Pin}(2)$ representation class. Then there exists $\gamma \in \Gamma$ such that $\gamma(x, y, z)$ is in one of the $S$-equivalence classes in Table 1.

The proof of Proposition 6.1 presented here is lengthy and highly computational. We begin by outlining the overall strategy. Consider all triples $(x, y, z)$ that arise from the solutions of Equation (2) provided by Theorem 5.4. For a triple $(x, y, z)$ to have a finite $\Gamma$-orbit, the four-term trigonometric equations that arise from repeated applications of $\tau_{X}$ or $\tau_{Y}$ must have solutions provided by Theorem 5.4 or violate the hypotheses of Theorem 5.4. We prove Proposition 6.1 by showing that all triples $(x, y, z)$ that arise from the solutions of Equation (2) provided by Theorem 5.4 fall into one of the following three categories:

1) $(x, y, z)$ has $k \geq 2$ or one of the global coordinates $(x, y, z)$ is zero.

2) $(x, y, z)$ belongs to one of the $S$-equivalence classes in Table 1 . Hence, corresponds to a $C$ or a $D$ representation class.

3) $(x, y, z)$ has infinite $\Gamma$-orbit with $-2<k<2$. Hence, has dense $\Gamma$-orbit by Proposition 4.3.

Definition 6.3. The pairs of angles $\left(\theta_{z}, \theta_{x}\right),\left(\pi-\theta_{x}, \pi-\theta_{z}\right)$, and $\left(\pi-\theta_{z}, \pi-\right.$ $\left.\theta_{x}\right)$ are called the symmetric, dual, and dual-symmetric pairs of $\left(\theta_{x}, \theta_{z}\right)$, respectively.

Recall that by a possible change of sign, each term in Equation (2) may be rewritten with angle in $[0, \pi / 2]$. Therefore, for fixed $a, b \in[0, \pi / 2]$, we 
obtain the following eight systems of equations

$$
\left\{\begin{array} { l } 
{ \operatorname { c o s } ( a ) = \pm \operatorname { c o s } ( \theta _ { x } + \theta _ { z } ) } \\
{ \operatorname { c o s } ( b ) = \pm \operatorname { c o s } ( \theta _ { x } - \theta _ { z } ) }
\end{array} \quad \left\{\begin{array}{l}
\cos (b)= \pm \cos \left(\theta_{x}+\theta_{z}\right) \\
\cos (a)= \pm \cos \left(\theta_{x}-\theta_{z}\right)
\end{array}\right.\right.
$$

for $\theta_{x}, \theta_{z} \in(0, \pi)$. The following, together with their dual, symmetric, and dual-symmetric pairs, are all possible pairs $\left(\theta_{x}, \theta_{z}\right)$ that satisfy one of the above eight systems of equations:

$$
\begin{gathered}
\left(\frac{a+b}{2},\left|\frac{a-b}{2}\right|\right),\left(\pi-\frac{a+b}{2},\left|\frac{a-b}{2}\right|\right), \\
\left(\pi / 2-\frac{a+b}{2}, \pi / 2+\frac{a-b}{2}\right),\left(\pi / 2-\frac{a+b}{2}, \pi / 2+\frac{b-a}{2}\right) .
\end{gathered}
$$

We prove in detail the cases in which Equation (2) corresponds to Equation 2 or 3 in Theorem 5.4. The argument for Equation 3 is the simplest and exemplifies the primary techniques used in the other cases. The case of Equation 2 involves a free parameter $t$, hence, is somewhat more involved than the others. For the other cases, we simply enumerate all the possible solutions and categorize them according to the above mentioned categories.

We first consider the case of

$$
\cos (\pi / 7)-\cos (2 \pi / 7)+\cos (3 \pi / 7)-\cos (\pi / 3)=0
$$

in detail. Consider $a=\pi / 7$ and $b=2 \pi / 7$. As given above, the possibilities for $\left(\theta_{x}, \theta_{z}\right)$ are

$$
(3 \pi / 14, \pi / 14),(11 \pi / 14, \pi / 14),(2 \pi / 7,3 \pi / 7),(2 \pi / 7,4 \pi / 7),
$$

along with their symmetric, dual, and dual-symmetric pairs. Suppose first that $\theta_{y}=\pi / 3$ (respectively, $2 \pi / 3$ ). Then the angles $\theta_{y} \pm \theta_{z}$ are rationally related to $\pi$ by reduced rationals with denominators 21 or 42 . Thus, for $(x, y, z)$ to have a finite $\Gamma$-orbit, the $\tau_{Y}$-equation at $\left(\theta_{x}, \theta_{y}, \theta_{z}\right)$ must correspond to Equation 1. Since $x \neq 0$ this equation can be rewritten as Equation 1 only if $y z-x=0$, or $y=\frac{x}{z}$. Then $y \neq \frac{x}{z}$ for $\theta_{y}=\pi / 3$ (or $2 \pi / 3$ ), and $x, z$ as given by the four pairs listed above (as well as their dual, symmetric, and dual-symmetric pairs). Thus the $\tau_{Y}$-equation at $\left(\theta_{x}, \theta_{y}, \theta_{z}\right)$ must violate the hypotheses of Theorem 5.4.

If $\theta_{x z-y}=\pi / 3$ or $2 \pi / 3$ then, consider the point $\tau_{X}\left(\theta_{x}, \theta_{y}, \theta_{z}\right)=$ $\left(\theta_{x}, \theta_{z}, \theta_{x z-y}\right)$ (in angle notation). The $\tau_{Y}$-equation at $\left(\theta_{x}, \theta_{z}, \theta_{x z-y}\right)$ is

$$
\cos \left(\theta_{z}+\theta_{x z-y}\right)+\cos \left(\theta_{z}-\theta_{x z-y}\right)-\cos \left(\theta_{x}\right)=\cos \left(\theta_{z(x z-y)-x}\right),
$$

but here $x z-y$ is playing the role of $y$ in the argument given above.

This type of argument works for the other five cases (e.g., $a=\pi / 7, \quad b=$ $3 \pi / 7$, etc.).

Now we cover the case

$$
\cos (t+\pi / 3)+\cos (\pi / 3-t)-\cos (t)=0 \quad(0<t<\pi / 6) .
$$


Recall that we are under the standing assumption $x, y, z \neq 0$. There are six cases.

Case 1: For $a=t+\pi / 3$, and $b=\pi / 3-t$, the possibilities are:

$$
(\pi / 3, t),(2 \pi / 3, t),(\pi / 6, \pi / 2+t),(\pi / 6, \pi / 2-t),
$$

and their dual, symmetric, and dual-symmetric pairs. Since $y \neq 0, \theta_{y}$ corresponds to $t$ and $x z-y=0$.

The first two pairs, together with their duals $(\pi / 3, \pi-t),(2 \pi / 3, \pi-t)$, yield $y= \pm z$. By Proposition 5.3, these triples are either $S$-equivalent to those appearing in Table 1 or have infinite $\Gamma$-orbits.

In the above symmetric (dual-symmetric) pairs, we have $y= \pm x$ and $z=$ 1. The $\tau_{X}$ preimage of $(x, \pm x, 1)$ is $\left(x, \pm x^{2}-1, \pm x\right)$ (signs taken together). Since $\pm x^{2}-1 \neq 0$, we may apply Proposition 5.3 to this triple as above. The same holds for $z=-1$.

Now consider the last two pairs. Note that the angle $\theta_{x}=\pi / 6$ does not appear in Equations 1-5. Therefore, any triple associated with these pairs will have an infinite $\Gamma$-orbit. For the symmetric pairs, i.e., $\theta_{z}=$ $\pi / 6$ (respectively, $5 \pi / 6$ ), note that $\tau_{X}\left(\theta_{x}, \theta_{y}, \theta_{z}\right)=\left(\theta_{x}, \theta_{z}, \theta_{x z-y}\right)$. The $\tau_{Y^{-}}$ equation at $\left(\theta_{x}, \theta_{z}, \theta_{x z-y}\right)$ cannot be put into the form of Equations 1-5, as above. This argument also applies to the duals of all such pairs.

Case 2: For $a=t+\pi / 3$, and $b=t$, the possibilities are:

$$
(t+\pi / 6, \pi / 6),(5 \pi / 6-t, \pi / 6),(\pi / 3-t, 2 \pi / 3),(\pi / 3-t, \pi / 3),
$$

and their dual, symmetric, and dual-symmetric pairs. Since $y \neq 0, \theta_{y}$ corresponds to $\pi / 3-t$ and $x z-y=0$.

As in the previous case, the last two pairs together with their dual, symmetric, and dual-symmetric pairs yield triples (or triples whose $\tau_{X}$ preimage) are either $S$-equivalent to those appearing in Table 1 or have infinite $\Gamma$-orbits. The argument for the first two pairs is also similar to that in the previous case.

Case 3: For $a=\pi / 3-t$, and $b=t$, the possibilities are:

$$
(\pi / 6, \pi / 6-t),(5 \pi / 6, \pi / 6-t),(\pi / 3,2 \pi / 3-t),(\pi / 3, t+\pi / 3),
$$

together with their dual, symmetric, and dual-symmetric pairs. An argument similar to the one given in case 1 holds.

Case 4: For $a=\pi / 3+t$, and $b=\pi / 2$, the possibilities are:

$$
\begin{aligned}
& (5 \pi / 12+t / 2, \pi / 12-t / 2),(7 \pi / 12-t / 2, \pi / 12-t / 2), \\
& (\pi / 12-t / 2,5 \pi / 12+t / 2),(\pi / 12-t / 2,7 \pi / 12-t / 2),
\end{aligned}
$$

and their duals. Note that the last two pairs are the symmetric pairs of the first two. As in previous arguments, it is enough to consider $\theta_{y}=t$ or $\pi-t$.

We first handle the triple (in angle notation) $(5 \pi / 12+t / 2, t, \pi / 12-t / 2)$. Note that three of the angles in the $\tau_{Y}$-equation of this point (all angles 
rewritten in $[0, \pi / 2])$ are: $5 \pi / 12+t / 2, \pi / 12+t / 2$, and $|\pi / 12-3 t / 2|$. However, both $|\pi / 12-3 t / 2|$ and $\pi / 12+t / 2$ correspond to angles in $[0, \pi / 6)$. If $\mid \pi / 12-$ $3 t / 2 \mid$ is nonzero, then the $\tau_{Y}$-equation of this point cannot correspond to Equations 1-5 since no equation in Theorem 5.4 has two angles in $[0, \pi / 6)$. If $|\pi / 12-3 t / 2|=0$, then Proposition 5.3 applies. For $(5 \pi / 12+t / 2, \pi-$ $t, \pi / 12-t / 2$ ), the angles in the $\tau_{Y}$-equation of this point (all angles rewritten in $[0, \pi / 2])$ are again: $5 \pi / 12+t / 2,|-\pi / 12+3 t / 2|$, and $\pi / 12+t / 2$.

The dual of the pair $(5 \pi / 12+t / 2, \pi / 12-t / 2)$ is $(7 \pi / 12-t / 2,11 \pi / 12+t / 2)$. Three of the angles in the $\tau_{Y}$-equation of $(7 \pi / 12-t / 2, t, 11 \pi / 12+t / 2)$ are: $7 \pi / 12-t / 2,11 \pi / 12+3 t / 2$, and $11 \pi / 12-t / 2$, which, when rewritten in [0, $\pi / 2]$, become: $5 \pi / 12+t / 2,|\pi / 12-3 t / 2|$, and $\pi / 12+t / 2$. These are the same angles as before, thus the same argument applies. The same argument also holds for $(7 \pi / 12-t / 2, \pi-t, 11 \pi / 12+t / 2)$.

Consider the symmetric pair $(\pi / 12-t / 2,5 \pi / 12+t / 2)$. For the triple $(\pi / 12-t / 2, t, 5 \pi / 12+t / 2)$, three of the angles in the $\tau_{Y}$-equation of this point are: $\pi / 12-t / 2,5 \pi / 12+3 t / 2$, and $5 \pi / 12-t / 2$. Note that the angle $5 \pi / 12+3 t / 2$, rewritten in $[0, \pi / 2]$, is:

$$
\begin{cases}5 \pi / 12+3 t / 2 & t \in(0, \pi / 18] \\ 7 \pi / 12-3 t / 2 & t \in[\pi / 18, \pi / 6) .\end{cases}
$$

This angle is in $(\pi / 3, \pi / 2]$. Further note that the angle $5 \pi / 12-t / 2$ is in $(\pi / 3, \pi / 2)$. A calculation rules out Equation 1. Now of the remaining equations, the angle $\pi / 12-t / 2$ can only correspond to the angle $\pi / 15$ in Equation 4. However, Equation 4 does not have any angles inside $(\pi / 3, \pi / 2)$. The same argument holds for the triple $(\pi / 12-t / 2, \pi-t, 5 \pi / 12+t / 2)$.

The dual of the pair $(\pi / 12-t / 2,5 \pi / 12+t / 2)$ is $(11 \pi / 12+t / 2,7 \pi / 12-$ $t / 2)$ and the $\tau_{Y}$-equation of the triples $(11 \pi / 12+t / 2, t, 7 \pi / 12-t / 2)$ and $(11 \pi / 12+t / 2, \pi-t, 7 \pi / 12-t / 2)$ have the same three angles (in $[0, \pi / 2])$ as above.

A similar argument holds for for the triples associated with the pairs $(7 \pi / 12-t / 2, \pi / 12-t / 2)$ and $(\pi / 12-t / 2,7 \pi / 12-t / 2)$.

Case 5: For $a=\pi / 3-t$, and $b=\pi / 2$, the possibilities are:

$$
\begin{aligned}
& (5 \pi / 12-t / 2, \pi / 12+t / 2),(7 \pi / 12+t / 2, \pi / 12+t / 2), \\
& (\pi / 12+t / 2,5 \pi / 12-t / 2),(\pi / 12+t / 2,7 \pi / 12+t / 2),
\end{aligned}
$$

and their duals. Note that the two last pairs are the symmetric pairs of the first two. As in previous arguments, it is enough to consider $\theta_{y}=t$ or $\pi-t$.

We first handle the triple (in angle notation) $(5 \pi / 12-t / 2, t, \pi / 12+t / 2)$. Note that three of the angles in the $\tau_{Y}$-equation of this point are: $5 \pi / 12-t / 2$, $\pi / 12+3 t / 2$, and $\pi / 12-t / 2$. A calculation shows that Equation 1 is ruled out. Thus, $\pi / 12-t / 2$ can only correspond to the angle $\pi / 15$ in Equation 4 , while $5 \pi / 12-t / 2$ is in $(\pi / 3, \pi / 2)$ which rules out Equation 4 . The same 
holds for the triple $(5 \pi / 12-t / 2, \pi-t, \pi / 12+t / 2)$ and those associated with the dual of $(5 \pi / 12-t / 2, \pi / 12+t / 2)$.

We now consider the triples associated with the symmetric pair $(\pi / 12+$ $t / 2,5 \pi / 12-t / 2)$. Consider the triple $(\pi / 12+t / 2, t, 5 \pi / 12-t / 2)$. Three of the angles in the $\tau_{Y}$-equation of this point are: $\pi / 12+t / 2,5 \pi / 12-3 t / 2$, and $5 \pi / 12+t / 2$. One can check that Equation 1 is ruled out. Now $5 \pi / 12+t / 2$ can only correspond to the angles $7 \pi / 15$ and $3 \pi / 7$ in Equations 3 and 5 . That is, $t=\pi / 10$ or $\pi / 42$. But in either case, the angle $\pi / 12+t / 2$ will not appear in any of Equations 2-5. The same holds for the triple $(\pi / 12+t / 2, \pi-$ $t, 5 \pi / 12-t / 2)$ and those associated with the dual of $(\pi / 12+t / 2,5 \pi / 12-t / 2)$.

Similar arguments hold for triples associated with the pairs $(7 \pi / 12+$ $t / 2, \pi / 12+t / 2)$ and $(\pi / 12+t / 2,7 \pi / 12+t / 2)$.

Case 6: For $a=t$, and $b=\pi / 2$, the possibilities are:

$$
\begin{aligned}
& (\pi / 4+t / 2, \pi / 4-t / 2),(3 \pi / 4-t / 2, \pi / 4-t / 2), \\
& (\pi / 4-t / 2, \pi / 4+t / 2),(\pi / 4-t / 2,3 \pi / 4-t / 2),
\end{aligned}
$$

and their duals. Note that the two last pairs are the symmetric pairs of the first two. As before, it is enough to consider $\theta_{y}=\pi / 3-t$ or $2 \pi / 3+t$.

We first handle the triple (in angle notation) $(\pi / 4+t / 2, \pi / 3-t, \pi / 4-t / 2)$. Note that three of the angles in the $\tau_{Y}$-equation are: $\pi / 4+t / 2,7 \pi / 12-3 t / 2$, and $\pi / 12-t / 2$. It is clear that Equation 1 is ruled out. The angle $\pi / 12-t / 2$ can only correspond to the angle $\pi / 15$ in Equation 4 , while $7 \pi / 12-3 t / 2$, when rewritten in $[0, \pi / 2]$, corresponds to an angle in $(\pi / 3, \pi / 2]$, which rules out Equation 4 . The same holds for the triple $(\pi / 4+t / 2,2 \pi / 3+t, \pi / 4-t / 2)$ and those associated with the dual of $(\pi / 4+t / 2, \pi / 4-t / 2)$.

Consider the symmetric pair $(\pi / 4-t / 2, \pi / 4+t / 2)$. For the triple $(\pi / 4-$ $t / 2, \pi / 3+t, \pi / 4+t / 2$ ), three of the angles (rewritten in $[0 \pi / 2]$ ) in the $\tau_{Y^{-}}$ equation are: $\pi / 4-t / 2, \pi / 12+t / 2$, and $5 \pi / 12-3 t / 2$. Note that both $\pi / 4-t / 2$ and $\pi / 12+t / 2$ are less than $\pi / 4$, leaving only Equations 1 and 4. However, a calculation rules out Equation 4, while Equation 1 is clearly ruled out. The same holds for the triple $(\pi / 4-t / 2,2 \pi / 3+t, \pi / 4+t / 2)$ and those associated with the dual of $(\pi / 4-t / 2, \pi / 4+t / 2)$.

Similar arguments hold for triples associated with the pairs $(3 \pi / 4-$ $t / 2, \pi / 4-t / 2)$ and $(\pi / 4-t / 2,3 \pi / 4-t / 2)$.

For the other three equations, we simply list the solutions $\left(\theta_{x}, \theta_{y}, \theta_{z}\right)$ in their respective category. For simplicity, we do not list the solutions correspond to the $\operatorname{Pin}(2)$ representations and those with one global coordinate equal to zero. Note that the complete set of solutions include the dual, symmetric, and dual-symmetric solutions in the first and third coordinates to those listed below.

$$
\cos (\pi / 5)-\cos (2 \pi / 5)-\cos (\pi / 3)=0
$$

1) $(k \geq 2)(\pi / 5, \pi / 3,3 \pi / 5),(\pi / 5,2 \pi / 3,2 \pi / 5)$. 
2) Triples appearing in Table $1:(\pi / 5,2 \pi / 3,3 \pi / 5),(\pi / 5, \pi / 3,2 \pi / 5)$.

3) Triples with an angle not appearing in Theorem 5.4:

$(3 \pi / 10, \pi / 3, \pi / 10),(3 \pi / 10,2 \pi / 3, \pi / 10),(7 \pi / 10, \pi / 3, \pi / 10)$,

$(7 \pi / 10,2 \pi / 3, \pi / 10),(7 \pi / 30,2 \pi / 5,13 \pi / 30),(7 \pi / 30,3 \pi / 5,13 \pi / 30)$,

$(7 \pi / 30,2 \pi / 5,17 \pi / 30),(7 \pi / 30,3 \pi / 5,17 \pi / 30),(7 \pi / 20,2 \pi / 5,3 \pi / 20)$,

$(7 \pi / 20,3 \pi / 5,3 \pi / 20),(7 \pi / 20, \pi / 3,3 \pi / 20),(7 \pi / 20,2 \pi / 3,3 \pi / 20)$,

$(3 \pi / 20,2 \pi / 5,13 \pi / 20),(3 \pi / 20,3 \pi / 5,13 \pi / 20),(3 \pi / 20, \pi / 3,13 \pi / 20)$,

$(3 \pi / 20,2 \pi / 3,13 \pi / 20),(11 \pi / 30, \pi / 5, \pi / 30),(11 \pi / 30,4 \pi / 5, \pi / 30)$,

$(19 \pi / 30, \pi / 5, \pi / 30),(19 \pi / 30,4 \pi / 5, \pi / 30),(9 \pi / 20, \pi / 5, \pi / 20)$,

$(9 \pi / 20,4 \pi / 5, \pi / 20),(9 \pi / 20, \pi / 3, \pi / 20),(9 \pi / 20,2 \pi / 3, \pi / 20)$,

$(11 \pi / 20, \pi / 5, \pi / 20),(11 \pi / 20,4 \pi / 5, \pi / 20),(11 \pi / 20, \pi / 3, \pi / 20)$,

$(11 \pi / 20,2 \pi / 3, \pi / 20),(5 \pi / 12, \pi / 5, \pi / 12),(5 \pi / 12,4 \pi / 5, \pi / 12)$,

$(5 \pi / 12,2 \pi / 5, \pi / 12),(5 \pi / 12,3 \pi / 5, \pi / 12),(7 \pi / 12, \pi / 5, \pi / 12)$,

$(7 \pi / 12,4 \pi / 5, \pi / 12),(7 \pi / 12,2 \pi / 5, \pi / 12),(7 \pi / 12,3 \pi / 5, \pi / 12)$.

Triples $(x, y, z)$ whose $\tau_{Y}$-equation does not correspond to any equation in Theorem 5.4:

$(4 \pi / 15,2 \pi / 5, \pi / 15),(4 \pi / 15,3 \pi / 5, \pi / 15),(11 \pi / 15,2 \pi / 5, \pi / 15)$, $(11 \pi / 15,3 \pi / 5, \pi / 15),(2 \pi / 15, \pi / 5,8 \pi / 15),(2 \pi / 15,4 \pi / 5,8 \pi / 15)$, $(2 \pi / 15, \pi / 5,7 \pi / 15),(2 \pi / 15,4 \pi / 5,7 \pi / 15)$.

Note that the categories above are not mutually exclusive.

$$
\cos (\pi / 5)-\cos (\pi / 15)+\cos (4 \pi / 15)-\cos (\pi / 3)=0 .
$$

1) $(k \geq 2)(\pi / 3,4 \pi / 5,2 \pi / 5),(3 \pi / 5, \pi / 5, \pi / 3)$.

2) Triples appearing in Table $1:(\pi / 3, \pi / 5,2 \pi / 5),(\pi / 3, \pi / 3,2 \pi / 5)$, $(\pi / 3,2 \pi / 3,2 \pi / 5),(3 \pi / 5,4 \pi / 5, \pi / 3),(3 \pi / 5, \pi / 3, \pi / 3)$.

3) Triples with an angle not appearing in Theorem 5.4:

$(11 \pi / 30,4 \pi / 15,17 \pi / 30),(11 \pi / 30,11 \pi / 15,17 \pi / 30)$,

$(11 \pi / 30, \pi / 3,17 \pi / 30),(11 \pi / 30,2 \pi / 3,17 \pi / 30)$,

$(11 \pi / 30,4 \pi / 15,13 \pi / 30),(11 \pi / 30,11 \pi / 15,13 \pi / 30)$,

$(11 \pi / 30, \pi / 3,13 \pi / 30),(11 \pi / 30,2 \pi / 3,13 \pi / 30),(7 \pi / 30, \pi / 15, \pi / 30)$,

$(7 \pi / 30,14 \pi / 15, \pi / 30),(7 \pi / 30, \pi / 3, \pi / 30),(7 \pi / 30,2 \pi / 3, \pi / 30)$,

$(23 \pi / 30, \pi / 15, \pi / 30),(23 \pi / 30,14 \pi / 15, \pi / 30),(23 \pi / 30, \pi / 3, \pi / 30)$,

$(23 \pi / 30,2 \pi / 3, \pi / 30),(7 \pi / 30, \pi / 15,13 \pi / 30)$,

$(7 \pi / 30,14 \pi / 15,13 \pi / 30),(7 \pi / 30,4 \pi / 15,13 \pi / 30)$,

$(7 \pi / 30,11 \pi / 15,13 \pi / 30),(7 \pi / 30, \pi / 15,17 \pi / 30)$,

$(7 \pi / 30,14 \pi / 15,17 \pi / 30),(7 \pi / 30,4 \pi / 15,17 \pi / 30)$,

$(7 \pi / 30,11 \pi / 15,17 \pi / 30),(\pi / 6, \pi / 5, \pi / 10),(\pi / 6,4 \pi / 5, \pi / 10)$,

$(\pi / 6, \pi / 3, \pi / 10),(\pi / 6,2 \pi / 3, \pi / 10)$,

$(5 \pi / 6, \pi / 5, \pi / 10),(5 \pi / 6,4 \pi / 5, \pi / 10)$,

$(5 \pi / 6, \pi / 3, \pi / 10),(5 \pi / 6,2 \pi / 3, \pi / 10),(3 \pi / 10, \pi / 5,11 \pi / 30)$,

$(3 \pi / 10,4 \pi / 5,11 \pi / 30),(3 \pi / 10,4 \pi / 15,11 \pi / 30)$,

$(3 \pi / 10,11 \pi / 15,11 \pi / 30),(3 \pi / 10, \pi / 5,19 \pi / 30)$,

$(3 \pi / 10,4 \pi / 5,19 \pi / 30),(3 \pi / 10,4 \pi / 15,19 \pi / 30)$, 
$(3 \pi / 10,11 \pi / 15,19 \pi / 30),(3 \pi / 10, \pi / 5, \pi / 30),(3 \pi / 10,4 \pi / 5, \pi / 30)$, $(3 \pi / 10, \pi / 15, \pi / 30),(3 \pi / 10,14 \pi / 15, \pi / 30),(7 \pi / 10, \pi / 5, \pi / 30)$, $(7 \pi / 10,4 \pi / 5, \pi / 30),(7 \pi / 10, \pi / 15, \pi / 30),(7 \pi / 10,14 \pi / 15, \pi / 30)$.

Triples $(x, y, z)$ whose $\tau_{Y}$-equation does not correspond to any equation in Theorem 5.4:

$(2 \pi / 15,4 \pi / 15, \pi / 15),(2 \pi / 15,11 \pi / 15, \pi / 15),(2 \pi / 15, \pi / 3, \pi / 15)$, $(2 \pi / 15,2 \pi / 3, \pi / 15),(13 \pi / 15,4 \pi / 15, \pi / 15),(13 \pi / 15,11 \pi / 15, \pi / 15)$, $(13 \pi / 15, \pi / 3, \pi / 15),(13 \pi / 15,2 \pi / 3, \pi / 15),(4 \pi / 15, \pi / 15,7 \pi / 15)$, $(4 \pi / 15,14 \pi / 15,7 \pi / 15),(4 \pi / 15, \pi / 3,7 \pi / 15),(4 \pi / 15,2 \pi / 3,7 \pi / 15)$, $(4 \pi / 15, \pi / 15,8 \pi / 15),(4 \pi / 15,14 \pi / 15,8 \pi / 15),(4 \pi / 15, \pi / 3,8 \pi / 15)$, $(4 \pi / 15,2 \pi / 3,8 \pi / 15),(\pi / 5, \pi / 15,7 \pi / 15),(\pi / 5,14 \pi / 15,7 \pi / 15)$, $(\pi / 5,4 \pi / 15,2 \pi / 15),(\pi / 5,11 \pi / 15,2 \pi / 15),(8 \pi / 15, \pi / 15, \pi / 5)$, $(4 \pi / 5,11 \pi / 15,2 \pi / 15),(4 \pi / 5,4 \pi / 15,2 \pi / 15),(8 \pi / 15,14 \pi / 15, \pi / 5)$. Triples with infinite orbits, by Proposition 5.3: $(\pi / 5, \pi / 5,2 \pi / 15),(\pi / 5,4 \pi / 5,2 \pi / 15),(4 \pi / 5, \pi / 5,2 \pi / 15)$, $(4 \pi / 5,4 \pi / 5,2 \pi / 15),(3 \pi / 5,2 \pi / 3, \pi / 3),(4 \pi / 15, \pi / 15, \pi / 15)$, $(4 \pi / 15,14 \pi / 15, \pi / 15),(4 \pi / 15,4 \pi / 15, \pi / 15),(4 \pi / 15,11 \pi / 15, \pi / 15)$, $(11 \pi / 15, \pi / 15, \pi / 15),(11 \pi / 15,14 \pi / 15, \pi / 15),(11 \pi / 15,4 \pi / 15, \pi / 15)$, $(11 \pi / 15,11 \pi / 15, \pi / 15),(\pi / 5, \pi / 5,7 \pi / 15),(\pi / 5,4 \pi / 5,7 \pi / 15)$, $(8 \pi / 15, \pi / 5, \pi / 5),(8 \pi / 15,4 \pi / 5, \pi / 5)$.

$$
-\cos (2 \pi / 5)+\cos (2 \pi / 15)-\cos (7 \pi / 15)-\cos (\pi / 3)=0 .
$$

1) $(k \geq 2)(\pi / 5,3 \pi / 5, \pi / 3),(\pi / 5,2 \pi / 3, \pi / 3),(2 \pi / 3,2 \pi / 5, \pi / 5)$, $(2 \pi / 3, \pi / 3, \pi / 5)$.

2) Triples appearing in Table $1:$

$(\pi / 5,2 \pi / 5, \pi / 3),(2 \pi / 3,2 \pi / 3, \pi / 5)(\pi / 5, \pi / 3, \pi / 3),(2 \pi / 3,3 \pi / 5, \pi / 5)$.

3) Triples with an angle not appearing in Theorem 5.4:

$(7 \pi / 30,7 \pi / 15,19 \pi / 30),(7 \pi / 30, \pi / 3,19 \pi / 30),(7 \pi / 30,8 \pi / 15,19 \pi / 30)$, $(7 \pi / 30,2 \pi / 3,19 \pi / 30),(7 \pi / 30,7 \pi / 15,11 \pi / 30),(7 \pi / 30, \pi / 3,11 \pi / 30)$, $(7 \pi / 30,8 \pi / 15,11 \pi / 30),(7 \pi / 30,2 \pi / 3,11 \pi / 30),(13 \pi / 30,2 \pi / 15, \pi / 30)$, $(13 \pi / 30, \pi / 3, \pi / 30),(13 \pi / 30,13 \pi / 15, \pi / 30),(13 \pi / 30,2 \pi / 3, \pi / 30)$, $(17 \pi / 30,2 \pi / 15, \pi / 30),(17 \pi / 30, \pi / 3, \pi / 30),(17 \pi / 30,13 \pi / 15, \pi / 30)$, $(17 \pi / 30,2 \pi / 3, \pi / 30),(11 \pi / 30,2 \pi / 15, \pi / 30),(11 \pi / 30,7 \pi / 15, \pi / 30)$, $(11 \pi / 30,13 \pi / 15, \pi / 30),(11 \pi / 30,8 \pi / 15, \pi / 30),(19 \pi / 30,2 \pi / 15, \pi / 30)$, $(19 \pi / 30,7 \pi / 15, \pi / 30),(19 \pi / 30,13 \pi / 15, \pi / 30),(19 \pi / 30,8 \pi / 15, \pi / 30)$, $(3 \pi / 10,2 \pi / 5, \pi / 6),(3 \pi / 10, \pi / 3, \pi / 6),(3 \pi / 10,3 \pi / 5, \pi / 6)$, $(3 \pi / 10,2 \pi / 3, \pi / 6),(7 \pi / 10,2 \pi / 5, \pi / 6),(7 \pi / 10, \pi / 3, \pi / 6)$, $(7 \pi / 10,3 \pi / 5, \pi / 6),(7 \pi / 10,2 \pi / 3, \pi / 6),(7 \pi / 30,2 \pi / 5, \pi / 10)$, $(7 \pi / 30,7 \pi / 15, \pi / 10),(7 \pi / 30,3 \pi / 5, \pi / 10),(7 \pi / 30,8 \pi / 15, \pi / 10)$, $(23 \pi / 30,2 \pi / 5, \pi / 10),(23 \pi / 30,7 \pi / 15, \pi / 10),(23 \pi / 30,3 \pi / 5, \pi / 10)$, $(23 \pi / 30,8 \pi / 15, \pi / 10),(\pi / 10,2 \pi / 5,17 \pi / 30),(\pi / 10,2 \pi / 15,17 \pi / 30)$, $(\pi / 10,3 \pi / 5,17 \pi / 30),(\pi / 10,13 \pi / 15,17 \pi / 30),(\pi / 10,2 \pi / 5,13 \pi / 30)$, $(\pi / 10,2 \pi / 15,13 \pi / 30),(\pi / 10,3 \pi / 5,13 \pi / 30),(\pi / 10,13 \pi / 15,13 \pi / 30)$. 
Triples $(x, y, z)$ whose $\tau_{Y}$-equation does not correspond to any equation in Theorem 5.4:

$(4 \pi / 15,7 \pi / 15,2 \pi / 15),(4 \pi / 15, \pi / 3,2 \pi / 15),(4 \pi / 15,8 \pi / 15,2 \pi / 15)$, $(4 \pi / 15,2 \pi / 3,2 \pi / 15),(11 \pi / 15,7 \pi / 15,2 \pi / 15),(11 \pi / 15, \pi / 3,2 \pi / 15)$, $(11 \pi / 15,8 \pi / 15,2 \pi / 15),(11 \pi / 15,2 \pi / 3,2 \pi / 15),(\pi / 15,2 \pi / 15,7 \pi / 15)$, $(\pi / 15, \pi / 3,7 \pi / 15),(\pi / 15,13 \pi / 15,7 \pi / 15),(\pi / 15,2 \pi / 3,7 \pi / 15)$, $(\pi / 15,2 \pi / 15,8 \pi / 15),(\pi / 15, \pi / 3,8 \pi / 15),(\pi / 15,13 \pi / 15,8 \pi / 15)$, $(\pi / 15,2 \pi / 3,8 \pi / 15),(4 \pi / 15,7 \pi / 15,2 \pi / 5),(4 \pi / 15,8 \pi / 15,2 \pi / 5)$, $(4 \pi / 15,7 \pi / 15,3 \pi / 5),(4 \pi / 15,8 \pi / 15,3 \pi / 5),(2 \pi / 5,2 \pi / 15, \pi / 15)$, $(2 \pi / 5,13 \pi / 15, \pi / 15),(3 \pi / 5,2 \pi / 15, \pi / 15),(3 \pi / 5,13 \pi / 15, \pi / 15)$.

Triples with infinite orbits, by Proposition 5.3:

$(4 \pi / 15,3 \pi / 5,3 \pi / 5),(4 \pi / 15,2 \pi / 5,3 \pi / 5),(3 \pi / 5,3 \pi / 5, \pi / 15)$, $(3 \pi / 5,2 \pi / 5, \pi / 15),(2 \pi / 5,3 \pi / 5, \pi / 15),(2 \pi / 5,2 \pi / 5, \pi / 15)$, $(2 \pi / 15,13 \pi / 15,8 \pi / 15),(2 \pi / 15,13 \pi / 15,7 \pi / 15),(4 \pi / 15,3 \pi / 5,2 \pi / 5)$, $(4 \pi / 15,2 \pi / 5,2 \pi / 5),(2 \pi / 15,2 \pi / 15,7 \pi / 15),(2 \pi / 15,2 \pi / 15,8 \pi / 15)$, $(2 \pi / 15,7 \pi / 15,7 \pi / 15),(2 \pi / 15,8 \pi / 15,7 \pi / 15),(2 \pi / 15,8 \pi / 15,8 \pi / 15)$, $(2 \pi / 15,7 \pi / 15,8 \pi / 15)$.

\section{References}

[1] J.H. Conway and A.J. Jones, Trigonometric diophantine equations (on vanishing sums of roots of unity), Acta Arithmetica, XXX (1976), 229-240.

[2] W.M. Goldman, Ergodic theory on moduli spaces, Ann. of Math., 146 (1997), 475-507.

[3] _ The symplectic nature of fundamental groups of surfaces, Adv. Math., 54 (1984), 200-225.

[4] B. Hasselblatt and A. Katok, Introduction to the Modern Theory of Dynamical Systems, Cambridge University Press, 1995.

[5] J. Huebschmann, Symplectic and Poisson Structures on Certain Moduli Spaces, preprint, hep-th/9312112, Pub. IRMA, Lille, 1993.

[6] D. Mumford and J. Fogarty, Geometric Invariant Theory, 2nd ed., Springer, Berlin, 1982.

[7] P.E. Newstead, Introductions to Moduli Problems and Orbit Spaces, Springer-Verlag, 1978.

Received September 2, 1998 and revised January 28, 1999.

Penn State Erie-The Behrend College

ERIE, PA 16563

E-mail address: jpp@vortex.bd.psu.edu

University of Massachusetts

AmHerst, MA 01003-4515

E-mail address: xia@math.umass.edu 